University of Nebraska - Lincoln

DigitalCommons@University of Nebraska - Lincoln

CSE Conference and Workshop Papers

Computer Science and Engineering, Department

2017

\title{
BioSIMP: Using Software Testing Techniques for Sampling and Inference in Biological Organisms
}

\author{
Mikaela Cashman \\ University of Nebraska-Lincoln, mcashman@cse.unl.edu \\ Jennie L. Catlett \\ University of Nebraska-Lincoln, jcatlett@acm.org \\ Myra B. Cohen \\ University of Nebraska-Lincoln, mcohen@iastate.edu \\ Nicole R. Buan \\ University of Nebraska-Lincoln, nbuan@unl.edu \\ Zahmeeth Sakkaff \\ University of Nebraska-Lincoln, zsayedsa@cse.unl.edu
}

See next page for additional authors

Follow this and additional works at: https://digitalcommons.unl.edu/cseconfwork

Part of the Bioinformatics Commons, Computer Engineering Commons, Electrical and Computer

Engineering Commons, Other Computer Sciences Commons, Research Methods in Life Sciences

Commons, and the Systems Biology Commons

Cashman, Mikaela; Catlett, Jennie L.; Cohen, Myra B.; Buan, Nicole R.; Sakkaff, Zahmeeth; Pierobon, Massimiliano; and Kelley, Christine A., "BioSIMP: Using Software Testing Techniques for Sampling and Inference in Biological Organisms" (2017). CSE Conference and Workshop Papers. 327.

https://digitalcommons.unl.edu/cseconfwork/327

This Article is brought to you for free and open access by the Computer Science and Engineering, Department of at DigitalCommons@University of Nebraska - Lincoln. It has been accepted for inclusion in CSE Conference and Workshop Papers by an authorized administrator of DigitalCommons@University of Nebraska - Lincoln. 


\section{Authors}

Mikaela Cashman, Jennie L. Catlett, Myra B. Cohen, Nicole R. Buan, Zahmeeth Sakkaff, Massimiliano Pierobon, and Christine A. Kelley 


\title{
BioSIMP:
}

\section{Using Software Testing Techniques for Sampling and Inference in Biological Organisms}

\author{
Mikaela Cashman, ${ }^{1}$ Jennie L. Catlett, ${ }^{2}$ \\ Myra B. Cohen, ${ }^{1}$ Nicole R. Buan, ${ }^{2}$ \\ Zahmeeth Sakkaff, ${ }^{1}$ Massimiliano Pierobon, ${ }^{2}$ \\ \& Christine A. Kelley ${ }^{3}$
}

1 Department of Computer Science \& Engineering, University of Nebraska-Lincoln Lincoln, NE, USA

2 Department of Biochemistry, University of Nebraska-Lincoln Lincoln, NE, USA

3 Department of Mathematics, University of Nebraska-Lincoln Lincoln, NE, USA

emails _mcashman@cse.unl.edu; jcatlett@acm.org; myra@cse.unl.edu; nbuan@unl.edu; zsayedsa@cse.unl.edu; pierobon@cse.unl.edu; ckelley2@math.unl.edu

\begin{abstract}
Years of research in software engineering have given us novel ways to reason about, test, and predict the behavior of complex software systems that contain hundreds of thousands of lines of code. Many of these techniques have been inspired by nature such as genetic algorithms, swarm intelligence, and ant colony optimization. In this paper we reverse the direction and present BioSIMP, a process that models and predicts the behavior of biological organisms to aid in the emerging field of systems biology. It utilizes techniques from testing and modeling of highly-configurable software systems. Using

Published in 2017 IEEE/ACM 12th International Workshop on Software Engineering for

Science (SE4Science)

DOI 10.1109/SE4Science.2017.9

Copyright (C) 2017 IEEE. Used by permission.
\end{abstract}


both experimental and simulation data we show that BioSIMP can find important environmental factors in two microbial organisms. However, we learn that in order to fully reason about the complexity of biological systems, we will need to extend existing or create new software engineering techniques.

Keywords: Systems Biology; Highly-Configurable Software

\section{Introduction}

Software engineering research has produced many techniques to reason about, test, and predict the behavior of complex software systems; systems which may have hundreds of thousands of lines of code and are highly-configurable. Highly-configurable software contains portions of code (features) that can be turned on or off in varying combinations. This feature-oriented view of software [1], [5] allows us to model, understand, and validate programs by considering common and variable code separately and by identifying sets of features which influence unique or undesirable behavior. Some of this research has turned to heuristics to sample large configuration spaces. Many of these sampling techniques are derived from biological phenomena such as genetic evolution, intelligent swarming, ant colony optimization, etc., since complex programs share traits with natural systems.

At the same time, recent work in systems biology aims to model and predict the behavior of organisms under specific environmental conditions (food sources, media composition, light, temperature, etc.) [13]. Many current efforts in systems biology utilize manually-curated models which have been meticulously modified by biologists to reflect current literature and experimental results [3]. Despite this effort, the models do not reflect the full capabilities of the organism being modeled. As a result, we are still unable to predict or control biological organisms well enough to leverage their full capabilities. Furthermore, faults in the design and failures in the predictions can have significant ecological and health consequences [19].

In this paper we utilize software testing techniques and develop a process to aid biologists in inferring and predicting behaviors in biological systems. We use software testing in order to systematically cover a large set of possible inputs, that result in a broad set of outputs. We relate biological organisms to highly-configurable software 
in which the underlying code of the organism is executed (or not) based on its environment (configurable options) leading to different observable behaviors. In biology the organism's code is its genotype while its observable behavior is its phenotype.

The contributions of this paper are twofold. First, we present a process called BioSIMP (Biological Sampling, Inference, Modeling, and Prediction), which samples, then tests and classifies the environmental inputs to infer influential factors, those factors which have an impact on the phenotypic outcomes, and to model the commonality and variability of the genomes leading to these behaviors. BioSIMP can then use the models for future predictions. Second, we perform a preliminary evaluation of BioSIMP on two real organisms extracted from the human gut, both in a laboratory setting and via simulation, and discover (some previously unknown) influential factors that impact growth.

\section{Motivation and Background}

Systems biology uses quantitative modeling to represent organisms as sets of interacting and communicating biochemical processes. We can view this as a source code level abstraction of the organism's behavior if we consider the organism itself as an executable program. The most common modeling approach is that of the genome-scale metabolic model which is based on a metabolic network [3], [7]. A metabolic network connects chemical reactions, and a chain of chemical reactions forms a pathway. The models are constructed through an iterative process that collects information from manually annotated genomes, known as pathway databases, inferences from similar organisms, and the body of literature to build a set of reaction equations and connect their flow [3]. This model can be used for detailed analysis of the metabolic potential of the organism using constraintbased modeling approaches such as Flux Balance Analysis [9], [11]. Flux Balance Analysis (FBA) is a widely used method to simulate the execution of these models. FBA takes environmental factors as inputs and uses a linear programming optimization methodology to compute the flow (flux) through the set of equations resulting in an output, for example the maximum growth. 


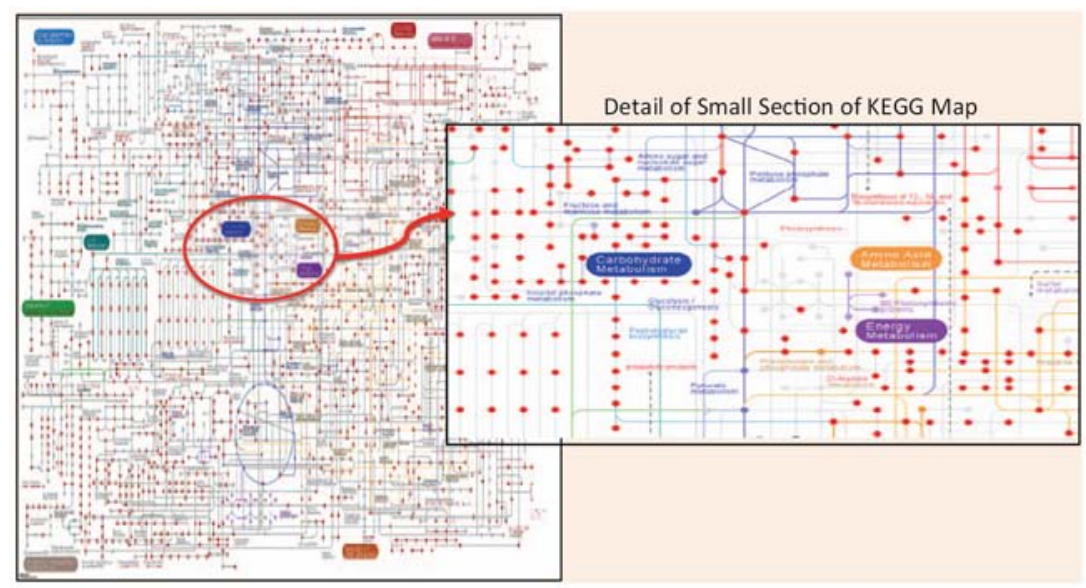

Fig. 1. Metabolic pathway map for Bacteroides thetaiotaomicron from the KEGG Database. On the left is the full map. On the right is a small detail.

Figure 1 shows a map of the metabolic model for one of the organisms that we use in our study, Bacteroides thetaiotaomicron (or $B$. theta) obtained from the Kyoto Encyclopedia of Genes and Genomes (KEGG) database [12]. The nodes are compounds that are inputs and outputs to the reactions (edges). As environmental inputs are utilized by the organism, a set of reactions creates paths through this network resulting in outputs (waste) to the environment, which can be used by other organisms. Similar bacterial networks contain more than 1000 reactions and 60,000 possible pathways [2].

As seen in Figure 1, a metabolic network derived from genomic information is too complex to analyze visually. We have expanded a small portion of the detail on the right portion of the figure. Trying to predict or understand the behavior of this organism by tracing the flow through all possible biochemical pathways would be like trying to manually extract meaning from a program with thousands of lines of code.

We also point out that these models are theoretical, and may not fully represent the real genomic model. Since they are created using limited experimental data, information from similar organisms are used to fill in the gaps. As a result some reactions and paths may be missing or infeasible. This has some similarity with a static model of software. Adding dynamic techniques (e.g. testing or laboratory experiments) allow us to iteratively refine the model. However, laboratory 
experimentation is labor-intensive and time-consuming (experiments for this paper took over a month), therefore we can only afford a limited number of dynamic runs.

\section{A. An Analogy to Configurable Software}

If we assume that the metabolic network is a model-based abstraction of our organism, then we can measure coverage of this model under differing environmental conditions. In the model, the reactions are the primitive elements. We can view these as statements within the code (and can measure reaction or code coverage) and evaluate the expected behavior (if available against the observed behavior), i.e. we can use software testing and map inputs (or configurations of the environment) to lines of code covered.

Since Figure 1 is too complex to understand visually, we present a running example in Figure 2 showing three examples (a-c) of a tiny section of the genome-scale metabolic model under different environmental conditions (representing compounds that are either present or not in the environment). In addition to a common set of compounds, the environment contains either Glucose, Hematin, or both Glucose and Hematin respectively. Each subfigure shows inputs (left) and outputs (right) for $B$. theta and the reactions through which those inputs and outputs flow. There are three reactions denoted as ovals (\#R38, \#R14 and \#R6). Reaction \#R38 (top) behaves identically in all

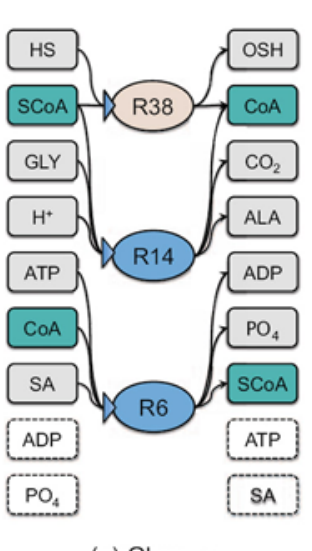

(a) Glucose

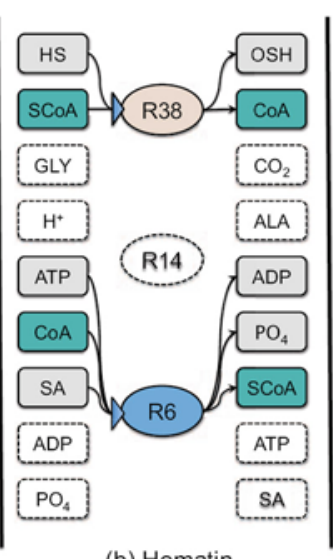

(b) Hematin

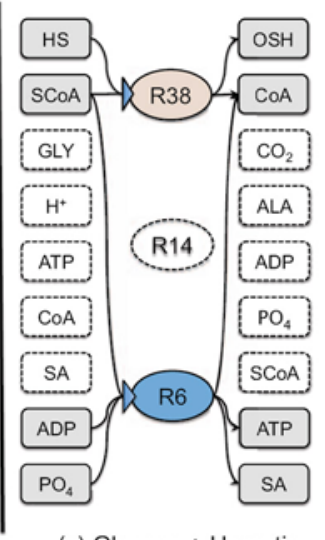

(c) Glucose + Hematin

Fig. 2. Reaction Paths in Different Environments 
three conditions, but the other two change. The shaded compounds (e.g. SA) are inputs to the reactions, while the unshaded, dashed compounds (e.g. ADP) are not contributing to any of these reactions under these conditions.

In the presence of Glucose only (Figure 2(a)), all three reactions occur. We observe two compounds (SCOA and CoA represented as darker shades) that appear both as inputs and outputs (the outputs can feed into another reaction that utilizes that compound). Figure 2(b) indicates that when the cells only have Hematin, reaction \#R14 (middle) is not covered during the program execution. However, the other two reactions behave similarly to when Glucose alone is in the culture medium. Finally, in Figure 2(c) we have a combination of Glucose and Hematin. Here we observe an unexpected pattern in the flow through the reactions. First, we again only observe two reactions that are executed in the model shown in Figure 2(c), despite the presence of both compounds (i.e. the reactions are not additive). Second, we see that \#R6 (bottom) behaves in the reverse direction under the combination of these factors (the inputs and outputs are switched). This real example suggests several things.

1) There is variability in how our code executes under differing environmental conditions.

2) There is some common behavior (e.g. reaction \#38).

3) The reactions can utilize different inputs and outputs under differing system configurations.

This behavior suggests that we can relate biological systems to highly-configurable software systems and perhaps leverage techniques from software testing and characterization to help infer their behavior and to determine which factors and/or combinations of factors are influential in changing behavior. For instance, in this exam-

ple both Glucose and Hematin, as well as their interaction, influence behavior.

\section{B. Sampling and Characterization}

Given the complexity of configurable software, techniques have been developed to sample and characterize faults during testing. Most real systems have too many configurations to enumerate and test. For 
instance the Linux kernel has been reported to have over 5,000 configuration options [17] leading to more than $2^{5000}$ configurations, while programs such as the GNU compiler, "gcc" have been reported to have as many as $10^{61}$ configurations [5].

One sampling technique that has been used extensively for sampling configurable software is combinatorial interaction testing (or CIT) [5], [22]. CIT samples broadly and systematically across factors (features) by generating small (optimized) samples that cover all $t$ way combinations of factors in at least one configuration. The variable $t$ is called the strength and determines how broadly we sample. When $t$ is 2 we call this pairwise sampling. The literature has shown that software testing is effective at lower strengths (2 or 3 ) [14]. One of the reasons we use CIT in our first phase of this work, is that it has been successfully combined with the use of classification trees for fault characterization to identify patterns of interactions among the features that lead to classes of faults [22]. Classification trees are heuristically produced with confidence levels, and guide the exploration of the identification of important factors [20].

Other sampling techniques have been proposed recently for performance testing [16], however we do not explore those sampling techniques in this work and leave the evaluation of alternative sampling techniques as future work.

\section{Related Work}

There has been some recent work on what is called executable biology [10], [21] where biological models are synthesized as finite state machines that describe their behavior and compared with laboratory data. This work does not identify influencing factors or attempt to identify variability. Other recent work on requirement analysis and model checking have been successfully applied to DNA self-assembly, in the context of fabricating nano-structures with processing capabilities [6], [15]. This research applies software engineering techniques to a biological structure (DNA), however their purpose is to program a single function for individual pathways at the nanolevel, while we are studying the complex system behavior of an entire organism as it interacts with its environment. 


\section{BioSIMP}

Figure 3 shows an overview of our process BioSIMP with each of the main steps: Biological Sampling, Inference, Modeling, and Prediction. Before using BioSIMP we initialize our system by identifying the features that will be varied within the environment. The choice of these depends on the biological organisms under study and requires some domain knowledge. For instance, in our experiments we have modeled the nutrient components of the microorganisms' culture medium. However, we could also model light or temperature, or levels of oxygen. In the first step \#(1) biological sampling selects configurations to test (this can be exhaustive in the case of a small model or use techniques such as CIT if the space is too large. We explore both options in our study). Experiments are then performed which involve executing the organisms' genomic software (code) under those configurations. This can be done in the laboratory, or via simulation (we use both in our study).

Once experiments are complete, we move to \#(2) inference. In this version of BioSIMP we use classification trees for characterization. We infer invariants at the behavioral (or phenomic) level. For example, we always see growth or a particular growth threshold under a particular combination of factors. We call these influential factors. Once identified, we model the influential factors \#(3) and map

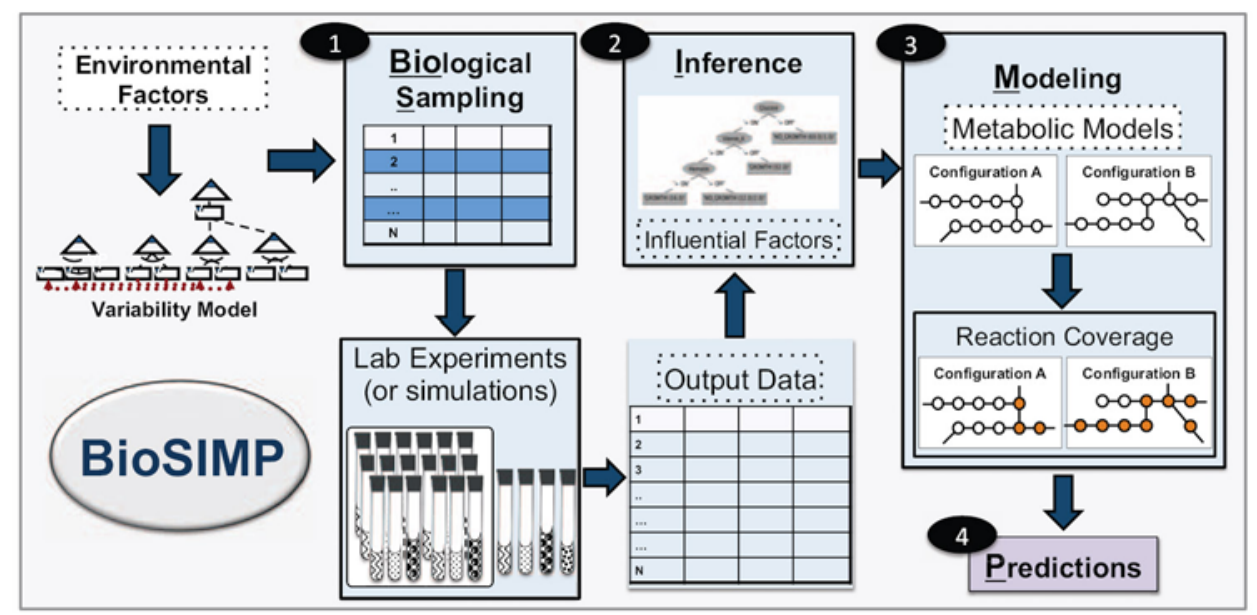

Fig. 3. The BioSIMP Process 
the behavioral invariants to code level invariants (i.e. we find the reactions coverage and fluxes). We perform this mapping using simulation (as we have done in our experiments) or by instrumenting the organisms with markers to identify particular intermediate outputs. We can identify the common and variable code which will allow us to focus only on the relevant code in the organisms' network model. This information can be used to iteratively focus lab experimentation on the important factors, to improve the existing models, and close the gap towards representing true organism behavior. Finally, we can use our new models \#(4) to predict the organisms' behavior in future scenarios.

The potential impact of BioSIMP is that it leads to simplified abstract models for understanding and predicting behavior in organisms by identifying influential factors without the need for exhaustive experimentation or manual curation.

\section{Case Study}

We performed a case study to evaluate the feasibility of using BioSIMP. We answer the following four research questions, one for each step in our process. ${ }^{1}$ To evaluate sampling we require inference, so we present inference first.

- RQ1 - Inference: Can BioSIMP identify influencing factors in practice?

- RQ2 - Sampling: How well does sampling work to identify the influencing factors?

- RQ3 - Modeling: Can we create a variability model for an organism?

- RQ4 - Prediction: Can we use BioSIMP to predict behavior and guide experiments?

1. Experimental artifacts and supplemental data can be found at: http://cse.unl.edu/ myra/artifacts/BioSIMP/ 


\section{A. Biological Systems Under Study}

We study two types of microbes found in humans: Bacteroides thetaiotaomicron (B. theta) and Methanobrevibacter smithii (M. smithii). The waste products of $B$. theta are hypothesized to be used by $M$. smithii in a synergy that benefits both microbes. Changes in the abundance of intestinal $B$. theta and $M$. smithii have been linked to nutrition-related disorders such as obesity [4]. Improved models of their interactions with each other and the human body would significantly enhance our knowledge of how gut microbes influence human health.

Independent Variables. We identified a set of nutritional compounds (environmental factors) based on the known requirements and products of each organism's metabolic system. These can be utilized as food, nutrients, or as toxins and are predicted to either encourage or hinder the an organism's growth. We chose only seven to make it feasible to obtain the ground truth by running the entire configuration space in the laboratory (the resource limiting factor). These include Glucose, Hematin, Formate, $H_{2^{\prime}}$ Vitamin $B_{12^{\prime}}$ Acetate, and Vitamin $K$. Each of these compounds can either be present in the solution (ON) or not (OFF). There is also a common set of compounds that all media contain. There are no known constraints on this model.

Dependent Variables. We utilize growth of the organisms in the given media as the dependent variable. Measuring growth of organisms in vitro is labor and resource-intensive, so we approximate by measuring optical density of microscale cultures, which is proportional to cell size and number. In silico, growth is determined by the sum of the flux (flow) through the biomass reactions using FBA techniques [9].

For RQ1 we generate classification trees to determine the influential factors. We evaluate the quality of the trees using both the accuracy and F-measure, two common metrics for this type of problem [20]. Accuracy gives the percentage the model correctly classified, while the F-measure is a balance between precision and recall. Precision calculates the number of true positives (TP), divided by the sum of true positives and false positives (FP): $T P \div(T P+F P)$. Recall measures the ratio of true positives, to the sum of true positives and false negatives (FN): $T P \div(T P+F N)$. The $\mathrm{F}$-measure is given as $2 R P \div(R+P)$ where $P$ is precision and $R$ is recall. We use the J48 classifier (an unpruned C4 decision tree) from Weka [20]. Classification trees require a training set to 
build the model, and a testing set to evaluate the model. For RQ1 we use the full configuration space for training, and a 10-fold cross validation. This type of cross validation has also been used on configurable software [22]. In RQ2 we use the sample data as our training set and evaluate the results against the full data.

For RQ3 we count the number of reactions common to all configurations, and the count that vary. This is obtained from the simulation data and uses the flux and its directions to differentiate coverage.

\section{B. Case Study Workflow}

We use both laboratory experimentation in vitro and simulations in silico using KBase, The Department of Energy Systems Biology Knowledgebase [11]. The experimental data provides us with dynamic, empirical evidence of the impact of the changing environmental factors for specific configurations. The simulation provides us with a (static) model that we can execute and enables us to collect the biochemical reactions that are executed under the selected configurations.

In vitro we create the 128 media and use them in the experiments described in Section IV-C. The lab provides us with a set of growth classes. In silico we begin by generating the 128 media files. These files are the input to our simulation system. KBase provides us with two outputs: reaction fluxes and growth class. The reaction fluxes are used in RQ3 to generate a reaction coverage model.

For RQ1 we use the growth classifications from all 128 configurations in vitro and then again for the in silico data. For RQ2 we created 30 CIT samples of each strength from 2-6 using the CASA tool [8]. We collect the same information for our analysis, but use only the sampled data to build the classification trees.

\section{Laboratory Experimentation}

The laboratory experiments consist of 128 media configurations in replicates of eight across 32 plates (96-wells each) before inoculation with either $B$. theta or M. smithii. After incubation, at a set period of time, the optical density is measured as an indication of growth. The experiment is repeated in triplicate for statistical relevance. The experiment required more than a month of human intensive time. 
Since the laboratory is open to human error, we use Chauvenet's criterion for data removal [18] to eliminate data that is likely to be spurious. To make the final determination of growth or no growth, we compare against 8 negative controls (media plates without any of the seven compounds, which lead to no growth). We use a high stringency statistical test (Equation 1) over the eight replicates to determine growth. If (1) is satisfied, growth is positive. Here OD is the optical density, STD is the standard deviation.

$$
O D\left(x_{i}\right)-\operatorname{STD}\left(x_{1}: x_{8}\right)>O D_{\text {avg }}\left(n_{1}: n_{8}\right)+2\left[S T D\left(n_{1}: n_{8}\right)\right]
$$

For each data point $x_{i}$ we compare its optical density minus the standard deviation of the 8 duplicates to the average optical density over the negative control's $\left(n_{1} \ldots n_{8}\right)$ duplicates. We provide some leniency by adding twice the standard deviation of the 8 duplicates back in. We use the mode of the 8 duplicates for each configuration as the result.

For $B$. theta we removed 56 of the 1024 data points as outliers based on Chauvenet's criterion. For M. smithii 52 of 1024 data points were removed. To avoid observer bias, combinations were removed from the analysis when three or more biological replicates of the eight were significantly different from the mode. Of the 128 combinations, seven from $B$. theta and 16 from M. smithii were removed.

\section{KBase Simulations}

KBase is an open source software hub and database designed for systems biology [11]. KBase combines data, tools, and results for predictive biology of microbes, plants, and their communities. KBase is also collaborative, allowing users to share data, create new automated analyses via a scripting language, and publish their results via narrative workflows.

For the simulation we ran a 3-step process. Step 1 is completed only once, while steps 2 and 3 are repeated for each configuration.

(1) Build Draft Model. Before we can simulate a growth experiment, we need to build the metabolic model starting with the genomes of B. theta and M. smithii provided in KBase. We use the Build Metabolic Model app in KBase which translates the organism's genome to protein sequences from a protein phylogeny database. This provides the initial model. 
(2) Gapfill Metabolic Model. In the first step, the model may be incomplete. The gapfilling algorithm [9] provides a way to fill in those missing links by adding known reactions from manual curation or from a global database to the model in order to force growth, if possible. We created the 128 different media configuration files and for each ran the Gapfill Metabolic Model app to obtain 128 Gapfilled Models.

(3) Flux Balance Analysis. We run each of the 128 gapfilled models through the Run Flux Balance Analysis (FBA) app to simulate the metabolites flowing through the organism's metabolic model. We use this information to build the reaction coverage model for RQ3. We use the biomass information to build the classification trees in RQ1 and RQ2.

\section{Results}

We now present the results of each research question.

\section{A. RQ1: Inference}

Figure 4 shows the classification trees for $B$. theta both in vitro (a) and in silico (b). The experimental data has two categories of outcomes (growth and no growth). The numbers in parentheses on each leaf are the tree splits for the data that has that configuration (left)

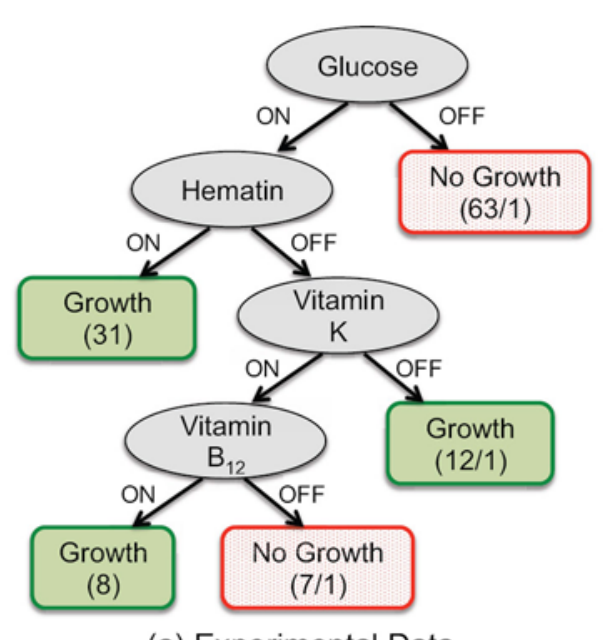

(a) Experimental Data

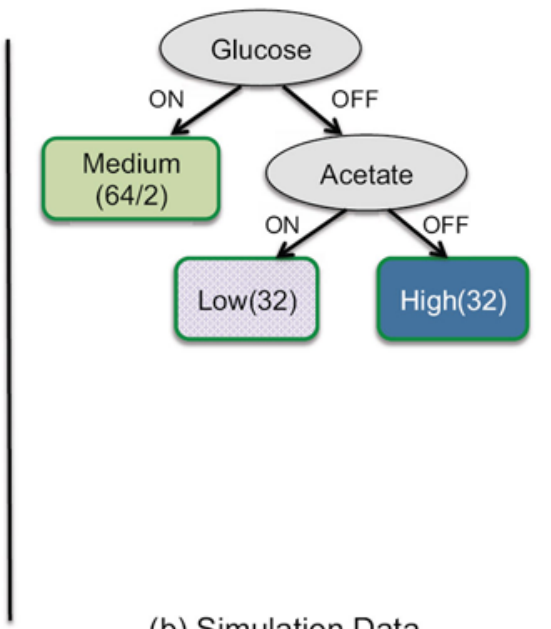

(b) Simulation Data

Fig. 4. B. theta Classification Trees 
or is mis-classified (right). The experimental tree has an overall accuracy of $97.52 \%$ and an F-measure of 0.98 . The primary split is on Glucose (62 of the 63 without Glucose did not grow) ${ }^{2}$, i.e. Glucose is required for growth. When Hematin is present in combination with Glucose there is growth. However when Hematin is not present the organisms grow without Vitamin K, or in the presence of both Vitamin $\mathrm{K}$ and Vitamin $\mathrm{B}_{12}$.

Figure 4(b) shows the tree for the simulation data. The tree is different from the experimental tree. First, the organism always grows based on the gapfilled metabolic models (see our discussion in Section $\mathrm{V}$-E). There are three distinct clusters of the predicted culture optical densities, therefore we use a tree with three output values (Low, Medium and High). The presence of Glucose leads to Medium growth. In its absence, the presence of Acetate leads to Low growth. Otherwise when neither Glucose nor Acetate is present there is High growth. This indicates the gapfill algorithm was able to find metabolic reactions in their absence that can lead to growth. The accuracy of this tree is $98.44 \%$ with an F-measure of 0.98 .

The M. smithii classification trees show similar results with an accuracy of $92.86 \%$ and an F-measure of 0.93 . M. smithii however, showed a more complex tree topology indicating that the factors interact in a more complex fashion. These can be found on our supplemental website.

Summary of RQ1. BioSIMP helps to interpret phenotype data and to identify relationships between influencing factors. Simulation only finds half of the influencing factors that are found in the lab, but also often finds alternative pathways through the organism. Without further analysis in the laboratory, we do not know if these pathways are feasible or not.

\section{B. RQ2: Sampling}

Our next question asks if we can use CIT to sample the configuration space to infer the same information at less cost. For each strength we show the results of $30 \mathrm{CIT}$ samples for the same model (due to the

2. The trees contain only the data kept after removal for experimental error, therefore the lab data may not add up to exactly 128 (see study description). 
B. theta Lab

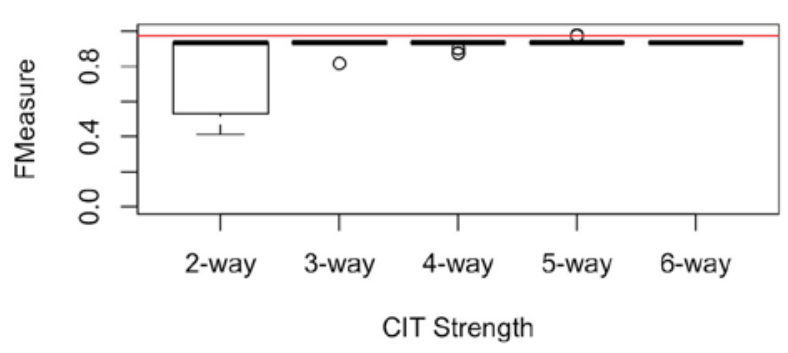

M. smithii Lab

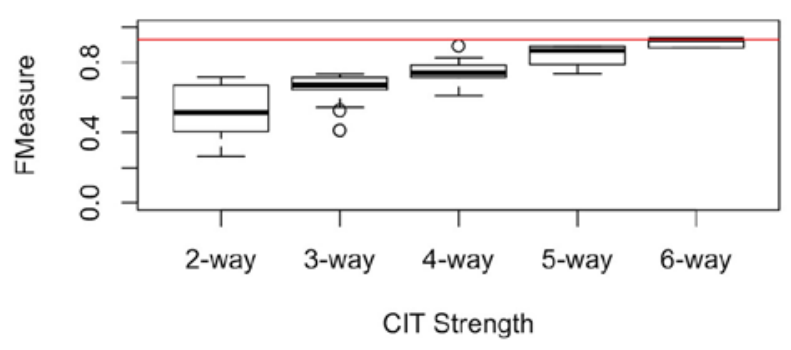

Fig. 5. F-measures by CIT Strength, for laboratory on B. theta and M. smithii. Data. Horizontal Line is the tree based on Exhaustive Analysis.

stochastic nature of (ASA). For each sample we show both the accuracy and F-measure. The results are averaged across samples (there is only one value for the full data set). Based on this data, Figure 5 shows boxplots for each strength CIT on $B$. theta and $M$. smithii in the laboratory. The simulation results are similar. In $B$. theta we would need to sample at strength 3 or higher to achieve an accuracy and F-measure above $90 \%$. In M. smithii however, we need to go as high as strength 5 or 6 to achieve the same result, again suggesting it is a more complex organism.

Summary of RQ2. CIT sampling is able to provide classification trees with good accuracy and F-measures, however, we need to sample at higher strengths (at least 3 for $B$. theta and 5 or 6 for M. smithii) than in traditional configurable software.

\section{RQ3: Modeling}

We next use KBase to study the variability in the reactions that are in the model for each of the configurations. We mark positive net flux as forward, negative net flux as reverse, and a zero net flux as unexecuted (uncovered). Table I shows detailed coverage data. A + indicates forward flow and a - indicates reverse flow. In the aggregate model for all configurations of $B$. theta there are 950 different reactions. $37.9 \%$ are common to all configurations. $29.5 \%$ have positive flow, $8.4 \%$ have a reverse flow and $39.8 \%$ are uncovered. 212 reactions have variable coverage depending on the influencing factors in the configuration. The range of total coverage is between 459 and 477 (48.3-50.2\%). We see a similar pattern for M. smithii. 
Table I. Reaction Coverage of Metabolic Model

B. theta (950 Reactions)

\begin{tabular}{lrr} 
& Count & Percent \\
\hline Common coverage+ & 280 & $29.5 \%$ \\
Common coverage- & 80 & $8.4 \%$ \\
Common Total & 360 & $37.9 \%$ \\
Uncovered & 378 & $39.9 \%$ \\
Variable coverage & 212 & $22.3 \%$ \\
Total Coverage Range: & $459-477$ & $48.3-50.2 \%$ \\
\hline M. smithii (908 Reactions) & & \\
& Count & \\
\hline Common coverage+ & 249 & Percent \\
Common coverage- & 79 & $27.4 \%$ \\
Common Total & 328 & $36.1 \%$ \\
Uncovered & 352 & $38.8 \%$ \\
Variable coverage & 228 & $25.1 \%$ \\
Total Coverage Range: & $430-448$ & $47.4-49.3 \%$ \\
\hline
\end{tabular}

We found 14 patterns of coverage in $B$. theta among the 128 configurations. These are shown in Figure 6 for a subset (37) of the reactions (complete data is available on our supplementary website). In this graph, the 14 configuration patterns are shown on the $y$-axis and the reaction number is shown on the $\mathrm{x}$-axis. A diamond represents forward (or positive flow) and an open circle represents reverse (or negative flow). The white space means uncovered. For easy reference the numbered reactions in our motivating example (\#6 and \#14) are highlighted in this figure. We do not see the other reaction (\#38) since it is part of the common coverage. As we can see the coverage pattern varies and as we often see in configurable software - we will either cover the reaction or not, depending on the configuration that is selected. The coverage model for $M$. smithii is similar and can be found on our website.

Summary of RQ3. We can obtain a dynamic variability model of the organism. In these organisms about one quarter (a little less than $26 \%$ ) of the reaction space varies. The implication is that we can target those reactions for further study. 


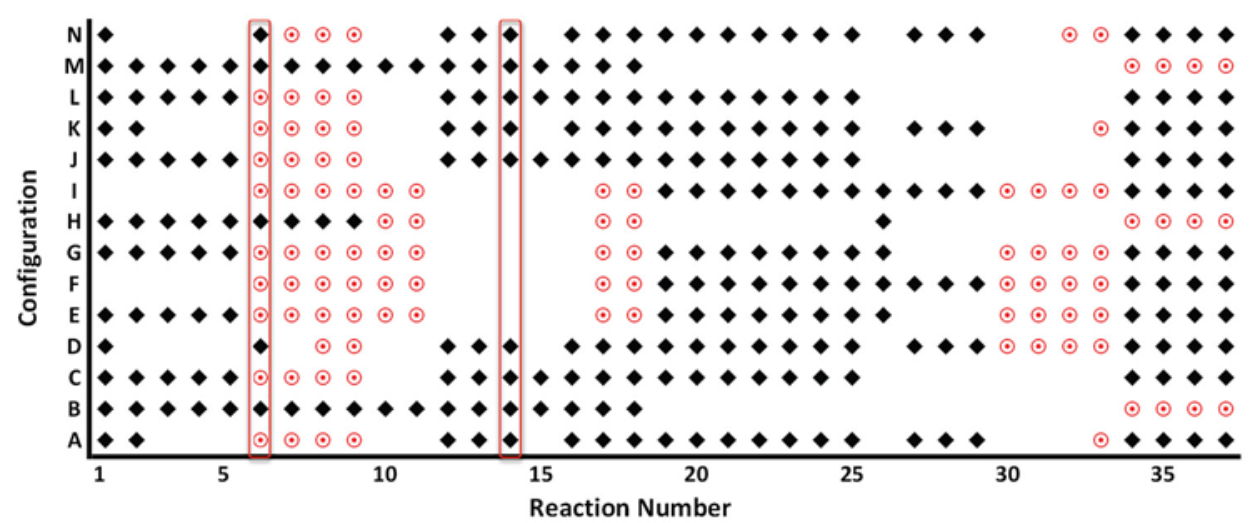

Fig. 6. Variable Coverage Model. Sample of 37 reactions in B. theta.

\section{RQ4: Prediction}

RQ4 asks if the inferences and models can assist biologists in simplifying the understanding of complex systems.

While not a formal user study, two co-authors anecdotally evaluated the classification trees and argue that they have proven very useful for sifting through the exhaustive phenotype datasets. The graphical trees clearly show which culture medium components resulted in growth and also suggest previously unknown positive and negative interactions between metabolites. These classifications hint at unknown gene regulatory networks and novel biochemical pathways that can be investigated through more invasive experiments. We discuss some learned observations next. (1) The trees predicted a previously unobserved interaction between Vitamin $\mathrm{K}$ and Vitamin $\mathrm{B}_{12}$. This has led to new experiments to explore its effect on $B$. theta and $M$. smithii. This has interesting implications for obesity, as a diet rich in foods such as fish and kale is high in Vitamin $\mathrm{K}$ and Vitamin $\mathrm{B}_{12}$. (2) BioSIMP shows that only $25 \%$ of the reactions shift in response to the available compounds. This suggests that a wider range of conditions are possible for the organisms than had previously been observed, and can be tested experimentally. (3) BioSIMP suggests 5-6 strength CIT will show $90 \%$ of the determinant factors for growth. While this is high for software testers, it reduces laboratory experiments by at least half in this study, a significant gain given the cost of experiments.

Summary of RQ4. The results obtained from BioSIMP are potentially useful for biologists who want to predict future behavior of an organism. 


\section{E. Discussion}

We have learned from this study that there are some fundamental differences and possible new directions for research in software engineering. First, we observed that the strength of interactions (3-6) is higher in biological systems than in software. Many of the algorithms for CIT are optimized for lower strength (2-3). This finding suggests that we will need higher strength algorithms for biological systems which will lead to stronger testing tools as well.

Second, we were not expecting bi-directional coverage of the model when we started. Although dataflow coverage in programs can indicate direction, element coverage such as statement or branch is usually counted as binary (covered or not). This brings up some interesting questions to explore, both on the biological side and on the software side.

Third, we discovered an interesting analogy that was not apparent at the start. The metabolic model design that is used is static. Hence the model has pathways that may or may not be feasible in practice. We do not know whether or not some of the pathways suggested by gapfilling algorithms are in fact feasible, however this is a question that we believe can be validated dynamically in the laboratory.

\section{Conclusions and Future Work}

In this paper we have presented a process BioSIMP that models environmental factors and then uses software testing techniques to sample, classify the results to infer influential features, and build models based on these inferences. This information can then be used to predict future behavior of biological systems.

In a case study on two human microbes, we show that BioSIMP is able to find influential factors in both the laboratory and in simulation. We also show that at most $26 \%$ of the reactions in the reaction network are variable allowing biologists to focus only on a narrow part of the network to understand behavior. We also note some differences with respect to software. First, we found that higher strength is needed in CIT sampling, and second we see that reaction coverage is not binary as is code coverage. We have seen that BioSIMP may 
help with hypothesis generation by identifying influential factors using both simulated and experimental data. This will eliminate wasted human effort and resources.

In future work we plan to explore alternative sampling techniques for BioSIMP and apply it to additional organisms. We also plan to study the new factors that we have identified more closely, and to evaluate the quality of our predictions and how we can use them more broadly. Finally, we plan to publish our KBase narratives for other scientists to use.

Acknowledgments - We would like to acknowledge Megan D. Smith for her help with some of the laboratory experimentation. This work is supported in part by NSF grants CCF-1161767, MCB-1449014 and IOS-1449525 and by NIH 1-P20GM113126-01. Any opinions, findings, and conclusions or recommendations expressed in this material are those of the authors and do not necessarily reflect the views of the funding agencies.

\section{References}

[1] S. Apel, D. Batory, C. Kastner, and G. Saake. Feature-Oriented Software Product Lines: Concepts and Implementation. Springer, 2013.

[2] M. Arita. The metabolic world of Escherichia coli is not small. In PNAS, volume 101, pages 1543-1547, 2004.

[3] G. J. E. Baart and D. E. Martens. Genome-scale metabolic models: reconstruction and analysis. Methods in Molecular Biology, 799:107- 126, 2012.

[4] F. Carbonero, A. C. Benefiel, and H. R. Gaskins. Contributions of the microbial hydrogen economy to colonic homeostasis. Nature reviews. Gastroenterology \& hepatology, 9(9):504-18, Sep 2012.

[5] M. B. Cohen, M. B. Dwyer, and J. Shi. Constructing interaction test suites for highly-configurable systems in the presence of constraints: A greedy approach. IEEE TSE, 34(5):633-650, 2008.

[6] S. J. Ellis, E. R. Henderson, T. H. Klinge, J. I. Lathrop, J. H. Lutz, R. R. Lutz, D. Mathur, and A. S. Miner. Automated requirements analysis for a molecular watchdog timer. In ASE, pages 767-778, 2014.

[7] K. Faust, D. Croes, and J. van Helden. Prediction of metabolic pathways from genome-scale metabolic networks. Elsevier, 2(105):109-121, 2011.

[8] B. J. Garvin, M. B. Cohen, and M. B. Dwyer. Evaluating improvements to a metaheuristic search for constrained interaction testing. EMSE, 16(1):61-102, 2011.

[9] C. S. Henry, M. DeJongh, A. A. Best, P. M. Frybarger, B. Linsay, and R. L. Sevens. High-throughput generation, optimization and analysis of genome-scale metabolic models. Nature Biotechnology, 28(9):977-982, August 2010. 
[10] N. P. Jasmin Fisher. Model Checking in Biology. Springer Verlag, January 2014.

[11] The Department of Energy Systems Biology Knowledgebase. http://kbase.us, 2016.

[12] Kyoto encyclopedia of genes and genomes. http://www.genome.jp/kegg/ , 2016.

[13] H. Kitano. Computational systems biology. Nature, 420(6912):206-10, 2002.

[14] D. R. Kuhn, D. R. Wallace, and A. M. Gallo. Software fault interactions and implications for software testing. IEEE TSE, 30(6):418-421, 2004.

[15] R. R. Lutz, J. H. Lutz, J. I. Lathrop, T. H. Klinge, D. Mathur, D. M. Stull, T. Bergquist, and E. R. Henderson. Requirements analysis for a product family of DNA nanodevices. In RE, pages 211-220, 2012.

[16] A. Sarkar, J. Guo, N. Siegmund, S. Apel, and K. Czarnecki. Cost-efficient sampling for performance prediction of configurable systems. In ASE, pages 342-352, 2015.

[17] S. She, R. Lotufo, T. Berger, A. Wąsowski, and K. Czarnecki. Variability model of the Linux kernel. In VaMoS, Linz, Austria, 2010.

[18] J. R. Taylor. An Introduction to Error Analysis The Study of Uncertainties in Physical Measurements. University Science Books, 1997.

[19] J. M. Tiedje, R. K. Colwell, Y. L. Grossman, R. E. Hodson, R. E. Lenski, R. N. Mack, and P. J. Regal. The Planned Introduction of Genetically Engineered Organisms: Ecological Considerations and Recommendations. Ecology, 70(2):298-315, Apr 1989.

[20] I. H. Witten, E. Frank, and M. A. Hall. Data Mining Practical Machine Learning Tools and Techniques. Elsevier, Burlington, MA, 2011.

[21] S. Woodhouse, N. Piterman, A. Koksal, and J. Fisher. Synthesizing Executable Gene Regulatory Networks from Single-Cell Gene Expression Data. Computer Aided Verification (CAV), pages 1-17, 2015.

[22] C. Yilmaz, M. B. Cohen, and A. Porter. Covering arrays for efficient fault characterization in complex configuration spaces. IEEE TSE, 31(1):20-34, Jan 2006. 